\title{
Evolving paradigms for successful molecular imaging of medullary thyroid carcinoma
}

\author{
Domenico Rubello $\cdot$ Ka Kit Wong • \\ Maria Cristina Marzola $\cdot$ Mohsen Beheshti • \\ Valentina Ambrosini • Sotirios Chondrogiannis • \\ Milton D. Gross
}

Published online: 5 January 2012

(C) Springer-Verlag 2011

Medullary thyroid cancer (MTC) is a rare, neuroendocrine tumour (NET) of parafollicular C cells comprising 5-8\% of all thyroid cancers with variable biological behaviour and prognosis [1]. Overall mean survival in MTC is $75-85 \%$ at 10 years [1-3]. Management of both hereditary (20-30\%) and sporadic MTC is challenging due to early cervical lymph node metastases occurring in $50 \%$ of patients and distant metastases to the liver, lung and bones being found in $10-20 \%$ at diagnosis $[1,3]$. MTC secretes several neuroendocrine peptides, of which calcitonin $(\mathrm{Ct})$ and less-specific carcinoembryonic antigen (CEA) are useful tumour markers for diagnosis, surveillance and prognosis. Yet despite the high sensitivity of an elevated serum $\mathrm{Ct}$ value as a predictor of disease presence, MTC is an elusive and notoriously problematic tumour to image [1, 3]. Anatomy-based imaging with CT and MRI has exhibited limited sensitivity due to the frequent small size of the metastatic

D. Rubello $(\bowtie) \cdot$ M. C. Marzola $\cdot$ S. Chondrogiannis Department of Nuclear Medicine, Medical Physics, Radiology, Santa Maria della Misericordia Hospital,

Via Tre Martiri 140,

45100 Rovigo, Italy

e-mail: domenico.rubello@libero.it

K. K. Wong $\cdot$ M. D. Gross

Department of Nuclear Medicine and Radiology,

University of Michigan Hospital,

Ann Arbor, MI, USA

M. Beheshti

PET-CT Center Linz, Nuclear Medicine and Endocrinology, Sant Vincent's Hospital,

Linz, Austria

V. Ambrosini

Department of Nuclear Medicine,

Policlinico Sant'Orsola-Malpighi, Bologna University, Bologna, Italy deposits. Although a variety of specific and nonspecific scintigraphic techniques such as ${ }^{201} \mathrm{Tl},{ }^{99 \mathrm{~m}} \mathrm{Tc}$-sestamibi, ${ }^{99 \mathrm{~m}} \mathrm{Tc}$-tetrofosmin, ${ }^{131} \mathrm{I} /{ }^{123} \mathrm{I}$-metaiodobenzylguanidine, ${ }^{99 \mathrm{~m}} \mathrm{Tc}-(\mathrm{V})$ dimercaptosuccinic acid (DMSA), and ${ }^{111} \mathrm{In}-$ octreotide have been used to image MTC, none has emerged as the imaging technique of choice $[4,5]$. This raises the question as to why we have been unable to successfully image MTC.

The rarity of MTC implies that no single centre can collect a sufficient number of cases, and to date there have been no large, cooperative studies to establish optimal imaging protocols. It has become apparent that MTCs are a heterogeneous group of neoplasms, and the biological behaviour of one MTC may differ significantly from that of another. Imaging approaches that have proven successful, for example using ${ }^{18} \mathrm{~F}$-fluorodeoxyglucose (FDG) PET/CT in lymphoma and solid cancers and ${ }^{111}$ In-octreotide for somatostatin receptor scintigraphy (SRS) in carcinoids, may be too simplistic an imaging approach for MTC, which often appears intermediate in biological behaviour between more aggressive neoplasms characterized by high Ki-67 indices, and hormonally active NETs in which FDG PET has limited value.

Genetic testing provides a valuable insight into the phenotypic expression of MTC. Genetic mapping has confirmed the central role of germline mutations of the rearranged during transfection (RET) protooncogene in the pathogenesis of inherited MTC and has shown that somatic RET mutations occur in $50-60 \%$ of patients with sporadic MTC, and there have been advances in our understanding of how activated RET tyrosine kinase signalling pathways affect tumour growth and angiogenesis [1, 6-8]. Insights from genetic testing allow informed management decisions. As an example, the dilemma of the timing of prophylactic thyroidectomy in multiple endocrine neoplasia type 2 (MEN2), an autosomal 
dominant syndrome with near-complete penetrance in $\mathrm{C}$ cell hyperplasia and evolution to MTC, has been somewhat clarified with the availability of risk stratification guidelines based upon mutation-specific classifications $[9,10]$. The American Thyroid Association (ATA) risk level D, including all MEN2B mutations, predicts an aggressive disease course with recommended early thyroidectomy before the age of 1 year. Conversely, for risk level A mutations, surgery may be safely delayed beyond the age of 5 years if basal and stimulated $\mathrm{Ct}$ levels remain normal.

In the last decade the success of FDG PET/CT in oncology imaging, and the increasing availability of hybrid PET/CT and SPECT/CT cameras, has fuelled intense interest in the development of multiple alternative single-photon-emitting somatostatin analogues and non-FDG positron-emitting radiopharmaceuticals, which demonstrate increased sensitivity for imaging MTC and NETs [5]. Total thyroidectomy with prophylactic central neck dissection, with or without lateral compartment dissection of lymph nodes is potentially curative in $60 \%$ of N0 patients, but in N1 patients cure rates fall to around $10 \%$ [3]. To our knowledge, there are no studies of the use of either PET/CT or SPECT/CT for preoperative MTC staging, although unlike radioiodine imaging in welldifferentiated thyroid cancer, total thyroidectomy is not a prerequisite for successful imaging of in-situ or locoregional metastatic disease in MTC. As recurrence rates of MTC approach $50 \%$, patients should be monitored after initial surgical management with $\mathrm{Ct}$ and CEA levels, including calculation of doubling times $[1,3,9,11]$. Should tumour biomarkers indicate persistent or recurrent disease, accurate restaging of MTC is crucial in subsequent management, whether to pursue a surgically based approach in an attempt to control locoregional disease, or to identify the best time to switch to systemic therapy for distant metastatic disease.

Hybrid SPECT/CT has superior diagnostic performance for endocrine imaging in comparison to the older techniques of planar imaging and SPECT. A PubMed search (22 October 2011) for reports on endocrine scintigraphy SPECT/ $\mathrm{CT}$ revealed more than 115 articles on radioiodine, metaiodobenzylguanine, iodomethyl-norcholesterol, somatostatin receptor and parathyroid scintigraphy, compared to only 10 articles at the end of 2005. SPECT/CT allows precise localization and characterization of radiotracer uptake (Fig. 1). To date there are no original studies concerning SPECT/CT imaging in MTC, probably reflecting the low incidence of this neoplasm, although a case report of a patient with paratracheal MTC recurrence characterized with ${ }^{111}$ In-octreotide SPECT/CT has been published [12], while another report of the detection of bone metastases in a patient with MEN2B using ${ }^{99 \mathrm{~m}} \mathrm{Tc}-(\mathrm{V}) \mathrm{DMSA}$ SPECT/CT [13]. Studies with newer somatostatin analogues such as ${ }^{99 \mathrm{~m}} \mathrm{Tc}-\mathrm{EDDA} /$ HYNIC-TOC have shown a sensitivity of $79.5 \%$ and a specificity of $83.3 \%$ for MTC [14], and further investigations of these radiopharmaceuticals should be performed with SPECT/CT when possible to ensure the highest diagnostic performance.

The first reported use of FDG PET in MTC was in 1997 where tumour was localized in $7 / 8$ patients $(88 \%)$ [15], while in a larger group of 85 patients with histological confirmation, FDG PET demonstrated a sensitivity $(78 \%)$ equal to that of MRI $(82 \%)$, and superior to those of CT $(50 \%)$, DMSA (33\%) and somatostatin scintigraphy $(25 \%)$ [16]. Two studies showed that FDG PET/CT outperforms $\mathrm{CT}$ and MRI for the detection of neck and mediastinal disease, but is inferior to CT for the detection of lung and liver metastases, and to MRI for the identification of metastases to bone $[17,18]$. Three studies have examined serum $\mathrm{Ct}$ levels and the likelihood of a positive FDG PET scan. In general a significant gain in sensitivity is seen at a threshold plasma Ct level of $>1,000 \mathrm{pg} / \mathrm{ml}$. Conversely, sensitivity is low at $0-37 \%$ when the plasma $\mathrm{Ct}$ level is $<500 \mathrm{pg} / \mathrm{ml}$ [19-21]. One study showed the prognostic value of FDG PET with 6 of 11 PET-positive patients (55\%) succumbing to their disease, and 13 of 14 PET-negative patients (93\%) remaining disease-free with an average follow-up of 44 months [22]. From these few studies it appears that FDG PET and PET/CT have an overall sensitivity in the range $47-79 \%$ with a trend to lower disease detection rates in more recent studies [5]. Although the use of FDG has been a significant step forward in the detection of MTC recurrences, it may be the optimal imaging technique for only a subset of patients with MTC.

Two novel positron-emitting radiopharmaceuticals, ${ }^{18} \mathrm{~F}$ fluorodihydroxyphenylalanine (FDOPA) and ${ }^{68} \mathrm{Ga}-\mathrm{DOTA}$ labelled somatostatin analogues, have shown promise in providing higher detectability of MTC lesions than FDG PET. FDOPA is an amino acid-derived radiotracer which is taken up, stored and decarboxylated by tumours belonging to the APUD system [23]. It was initially developed for neurological scanning [24, 25]. In 2001 Hoegerle et al. compared the value of FDOPA PET with that of FDG PET, SRS and morphological tomography imaging (i.e. CT, MRI) in patients with MTC and elevated Ct levels [26]. The sensitivity of FDOPA PET (63\%) was superior to those of FDG PET (44\%) and SRS (52\%) for detection of MTC lesions. Although the sensitivity of morphological imaging modalities was higher than that of functional imaging, the authors concluded that neither FDOPA nor morphological imaging alone achieves sufficient sensitivity and specificity to be used as a sole imaging modality in the assessment of patients with MTC. Among the functional imaging modalities, the superiority of FDOPA PET (Fig. 2) has been shown by several studies with a sensitivity ranging from $44 \%$ to $90 \%$ [26-32]. The results of these studies have also been confirmed in the preclinical setting: FDOPA small-animal PET was also found to 
Fig. 1 Postsurgical diagnostic $37 \mathrm{MBq}{ }^{131}$ I SPECT/CT scan in a 49-year-old woman with an 8-mm papillary thyroid microcarcinoma. a Anterior static neck and thorax image demonstrates focal uptake in the thyroid bed (arrow). b, c Axial SPECT (b) and CT coregistered (c) images show a $10-\mathrm{mm}$ pretracheal lymph node which is not iodine-avid (localizer). d-f Axial SPECT (d), CT (e) and fused SPECT/CT (f) images show neck uptake in the left thyroid bed compatible with remnant thyroid tissue after surgery (localizer). The noniodine avid level VI pretracheal lymph node was subsequently confirmed as metastatic thyroid cancer on ultrasound-guided biopsy. Histology was consistent with MTC after surgical resection. Review of the initial surgical specimen detected a tiny $(2-3 \mathrm{~mm})$ focus of primary MTC in the opposite lobe to the original papillary thyroid cancer
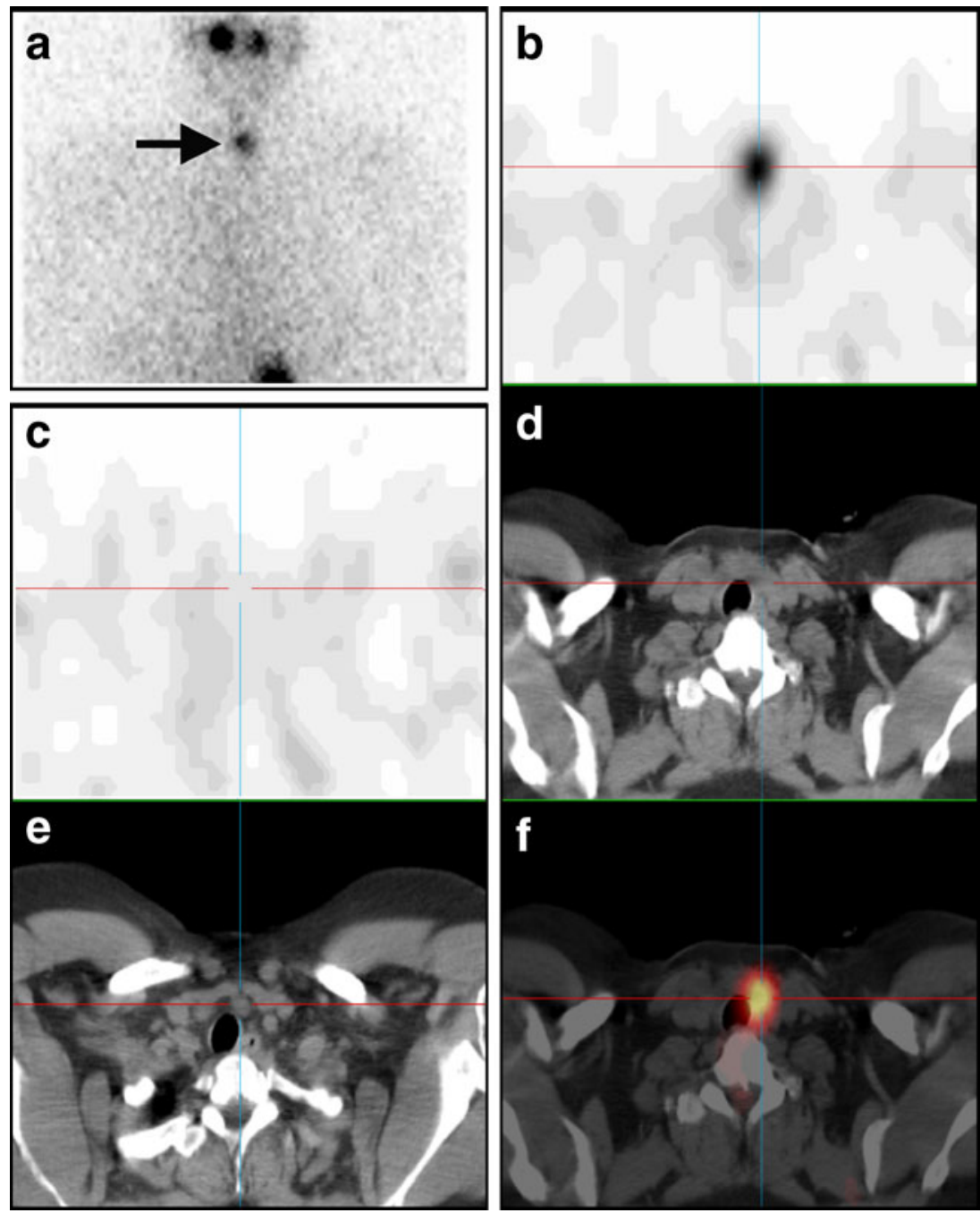

be accurate for monitoring disease progression over time [33].

In a recent study, Luster et al. [34] found that no patient with a basal $\mathrm{Ct}$ level less than $60 \mathrm{pg} / \mathrm{ml}$ was truly positive for MTC on FDOPA PET/CT, and conversely that no patient with a basal $\mathrm{Ct}$ level more than $120 \mathrm{pg} / \mathrm{ml}$ was truly negative for MTC on PET/CT. They reported a sensitivity and specificity of $100 \%$ for FDOPA PET/CT in patients with a basal

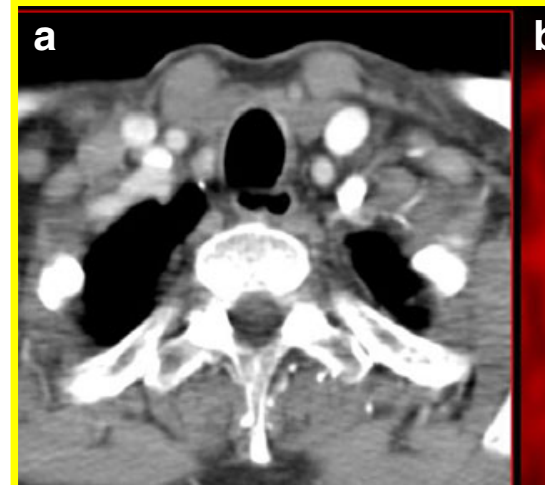

CT b

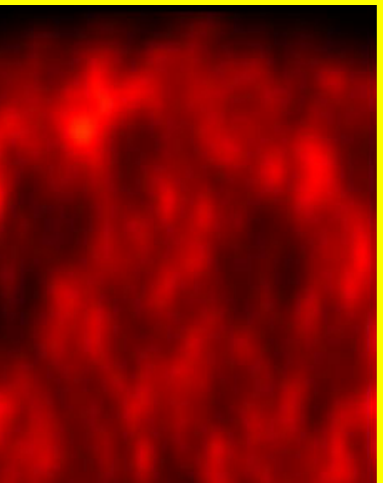

FDG

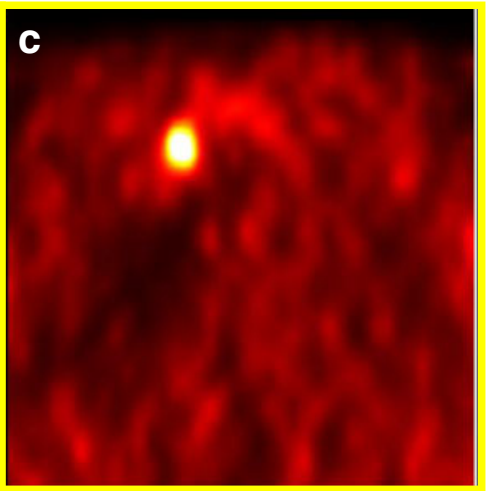

F-DOPA
Fig. 2 A 70-year-old patient with MTC and elevated tumour markers after total thyroidectomy (Ct $180 \mathrm{pg} / \mathrm{ml}$, CEA $1.5 \mathrm{ng} / \mathrm{ml})$. a Axial CT image of the thyroid bed. b Axial FDG PET image is negative. c Axial
FDOPA PET image shows an area of intense tracer uptake in the right paratracheal region corresponding to MTC relapse 
Ct level of $\geq 150 \mathrm{pg} / \mathrm{ml}$. The findings of this study were in line with the 2009 American Thyroid Association MTC management guidelines [9] that suggest additional imaging procedures (e.g. FDOPA PET/CT) should be applied to supplement cervical ultrasonography in any patient with a postsurgical basal Ct level of $\geq 150 \mathrm{pg} / \mathrm{ml}$. Similar results were also obtained in a very recent prospective study by Kauhanen et al. [35] who compared FDOPA and FDG PET/ CT with multidetector CT (MDCT) and MRI in recurrent or persistent MTC. They concluded that FDOPA PET/CT and FDG PET/CT were complementary in patients with an unstable $\mathrm{Ct}$ level; however, in patients with an unstable CEA doubling time, FDG PET/CT seemed to be the preferred option. In this study, MRI proved to be a sensitive method for detection of occult MTC lesions, but it mainly suffered from inadequate specificity as it had the highest rate of false-positive lesions. It is worth noting that in a very recent ongoing study, Marzola et al. [36] evaluated 18 MTC patients with rapidly increasing serum $\mathrm{Ct}$ levels and negative conventional imaging after first treatment, comparing the results of FDG PET/CT and those of FDOPA PET/CT. These authors also found that FDG and FDOPA can play a complementary role, but they also reported that in three patients FDOPA was the only positive examination for solitary local relapse in the thyroid bed and that prompt reoperation led to normalization of the serum $\mathrm{Ct}$ levels in two patients. So the authors concluded that FDOPA PET/CT seems able to detect local MTC relapse early in a phase when the patient can be successfully cured by reoperation.

While most studies have shown clear superiority of FDOPA PET/CT over FDG PET/CT [27, 30, 35], it is assumed that the combination of the two radiopharmaceuticals would probably provide the highest diagnostic sensitivity and specificity [34-35], in particular in patients with short ( $<6$ months) Ct doubling times [27]. FDOPA seems to provide more reliable prognostic information whereas FDG allows prognostic evaluation [36].

To our knowledge there is only a single published study specifically aimed at assessing the role of PET/CT with ${ }^{68} \mathrm{Ga}$-DOTA peptides in MTC. The authors compared the accuracy of ${ }^{68} \mathrm{Ga}$-DOTATATE PET/CT and ${ }^{18} \mathrm{~F}$-FDG PET/ $\mathrm{CT}$ in 18 patients previously radically treated for MTC and showing increasing serum Ct levels [27]. Both tracers showed similar suboptimal sensitivity values $(72.2 \%$ for DOTATATE, $77.8 \%$ for FDG) for the detection of recurrent MTC. The observation that ${ }^{68} \mathrm{Ga}$-DOTA peptide PET/CT may be useful in MTC patients but may fail to delineate either the whole tumour burden or tumour lesions with variable expression of somatostatin receptors has also been shown in single MTC cases described in the literature in the context of a large population of patients with NET with the location of the primary tumour other than in the thyroid. For example, of the only two patients with MTC among a population of 51 patients with NET with a nonthyroid primary, DOTATATE uptake was evident in one of the patients with an intermediate differentiation grade and absent in the other patient with a low differentiation grade [37]. The most relevant factor limiting MTC imaging with ${ }^{68} \mathrm{Ga}$-DOTA peptides is the well-known lower incidence, variable density and an inhomogeneous pattern of somatostatin receptor expression on MTC cells [38, 39]. Therefore the effective clinical impact of ${ }^{68} \mathrm{Ga}$-DOTA peptides in the diagnosis of MTC lesions remains controversial. However, it is important to underline that the confirmation of somatostatin receptors on MTC lesions in patients positive on ${ }^{68} \mathrm{Ga}$-DOTA peptide PET may provide the indication to start radionuclide-targeted therapy that has been reported to be associated with a longterm survival benefit [40]. ${ }^{68} \mathrm{Ga}$-DOTA peptide PET positivity would suggest an increased likelihood of the efficacy of ${ }^{177} \mathrm{Lu}$ - or ${ }^{90}$ Y-labelled somatostatin analogue radionuclide therapy.

${ }^{11} \mathrm{C}$-methionine PET has also been used to image MTC, although the reported lesion-based sensitivity of $73 \%$ is similar to that of FDG PET $(80 \%)$, and thus provides no clear advantage over FDG PET [41].

Unlike initial surgical management, reoperation for recurrent MTC rarely normalizes serum Ct levels [42] and the availability of more sensitive radiopharmaceuticals for localizing distant metastatic spread would reduce morbidity related to furtive surgical management. The lack of efficacy of external beam radiation treatment [3, 43, 44], the low chemotherapy response rates of only $10-20 \%[1,45,46]$, and the modest impact of ${ }^{131} \mathrm{I}$ - or ${ }^{90} \mathrm{Y}$-labelled targeted radionuclide therapies with response rates of approximately $30 \%$ [45], provide great impetus for studies of novel multikinase inhibitors as new approaches to therapy $[45,46]$. The present clinical guidelines recommend that kinase inhibitors are the preferred best "current practice" therapy for inoperable, progressive MTC and should be investigated in clinical trials $[6,9,11]$. These small-molecule inhibitors, which include vandetanib and sorafenib, block ATP at catalytic sites in the RET tyrosine kinase activation pathway and have been found to have modest efficacy for stabilization of aggressive disease [6-8, 47]. Vandetanib is the first kinase inhibitor, approved by the FDA in April 2011 [6, 48], and is generally well tolerated although cardiovascular, dermatological, endocrine and gastrointestinal toxicities have been encountered [7].

Eligibility for trials of kinase inhibitors is currently based on demonstrable macroscopic disease amenable to RECIST anatomical response assessment [8]. However, some patients with elevated and rapidly rising $\mathrm{Ct}$ levels, in whom the source(s) cannot be identified will fail to meet inclusion criteria. Reliance on decreasing tumour size as a marker of response fails to account for the presence of amyloid sheets, found in $60-80 \%$ of MTC tumours, which may persist 
despite a therapeutic response, so that drug efficacy is underestimated $[1,8,43]$. Recently published PERCIST criteria highlight the importance of including metabolic response criteria into clinical trials of anticancer drugs, as these are more closely correlated with therapeutic response and survival [48]. Metabolic response criteria using FDG PET in mouse models for predicting early therapeutic efficacy of vandetanib have been reported [49]. Resistance to kinase inhibitors, recognized as arising from tumour activation of alternative signalling pathways [7], appears to account for decreased drug effect(s), and molecular imaging techniques may more reliably distinguish responders and nonresponders.

Management of MTC requires highly accurate staging and restaging imaging techniques to optimize treatment strategies. For determination of a surveillance strategy for both sporadic and hereditary MTC, we now have the toolset to characterize tumours. Genetic testing, histological differentiation and grade (Ki-67 indices) [31], tumour secretogogues, and $\mathrm{Ct}$ and CEA levels and doubling times [1,9] can be used to develop a phenotype model to tailor individualized treatment and imaging surveillance. Customized imaging approaches will determine which radiopharmaceutical (FDG or non-FDG, especially FDOPA) is optimal for PET imaging of a given patient with MTC. Conversely, these imaging studies will provide unique information on individual tumour phenotype expression, including lack of tumour avidity, which may reveal as much about tumour behaviour and prognosis as a positive study.

Thus, our early experience suggests that molecular imaging will play an increasingly important role in the development of successful imaging and treatment strategies in MTC.

\section{Conflicts of interest None.}

\section{References}

1. Cohen EG, Shaha AR, Rinaldo A, Devaney KO, Ferlito A. Medullary thyroid carcinoma. Acta Otolaryngol. 2004;124:544-57.

2. Roman S, Mehta P, Sosa JA. Medullary thyroid cancer: early detection and novel treatments. Curr Opin Oncol. 2009;21:510.

3. Wu LS, Roman SA, Sosa JA. Medullary thyroid cancer: an update of new guidelines and recent developments. Curr Opin Oncol. 2011;23:22-7.

4. Rufini V, Castaldi P, Treglia G, Perotti G, Gross MD, Al-Nahhas A, et al. Nuclear medicine procedures in the diagnosis and therapy of medullary thyroid carcinoma. Biomed Pharmacother. 2008;62:13946.

5. Wong KK, Arabi M, Zerizer I, Al-Nahhas A, Rubello D, Gross MD. Role of positron emission tomography/computed tomography in adrenal and neuroendocrine tumors: fluorodeoxyglucose and nonfluorodeoxyglucose tracers. Nucl Med Commun. 2011;32:764-81.
6. Gild ML, Bullock M, Robinson BG, Clifton-Bligh R. Multikinase inhibitors: a new option for the treatment of thyroid cancer. Nat Rev Endocrinol. 2011;7:617-24.

7. Prazeres H, Torres J, Rodrigues F, Couto JP, Vinagre J, SobrinhoSimoes M, et al. How to treat a signal? Current basis for RETgenotype-oriented choice of kinase inhibitors for the treatment of medullary thyroid cancer. J Thyroid Res. 2011;2011:678357.

8. Ye L, Santarpia L, Gagel RF. The evolving field of tyrosine kinase inhibitors in the treatment of endocrine tumors. Endocr Rev. 2010;31:578-99.

9. Kloos RT, Eng C, Evans DB, Francis GL, Gagel RF, Gharib H, et al. Medullary thyroid cancer: management guidelines of the American Thyroid Association. Thyroid. 2009;19:565-612.

10. Grubbs EG, Waguespack SG, Rich TA, Xing Y, Ying AK, Evans DB, et al. Do the recent American Thyroid Association (ATA) Guidelines accurately guide the timing of prophylactic thyroidectomy in MEN2A? Surgery. 2010;148:1302-9. discussion 1309-10.

11. Tuttle RM, Ball DW, Byrd D, Daniels GH, Dilawari RA, Doherty GM, et al. Medullary carcinoma. J Natl Compr Canc Netw. 2010;8:512-30.

12. Fiore D, Rubello D, Casara D, Pelizzo MR, Franchi A, Muzzio PC. Role of CT/111In-octreotide SPECT digital fusion imaging in the localization of loco-regional recurrence of medullary thyroid carcinoma. Minerva Chir. 2004;59:295-9.

13. De Bonilla-Damia A, Calvo-Moron C, De La Riva-Perez PA, Iglesias-Jerez R, Molina-Mora M, Castro-Montano J. Detection by SPECT-CT scan with $(99 \mathrm{~m}) \mathrm{Tc}-(\mathrm{V})$ DMSA of bone metastases in patient with medullary thyroid cancer. Rev Esp Med Nucl. 2011;30:365-7.

14. Czepczynski R, Parisella MG, Kosowicz J, Mikolajczak R, Ziemnicka $\mathrm{K}$, Gryczynska M, et al. Somatostatin receptor scintigraphy using 99mTc-EDDA/HYNIC-TOC in patients with medullary thyroid carcinoma. Eur J Nucl Med Mol Imaging. 2007;34:1635-45.

15. Adams S, Baum RP, Hertel A, Schumm-Draeger PM, Usadel KH, Hor G. Comparison of metabolic and receptor imaging in recurrent medullary thyroid carcinoma with histopathological findings. Eur J Nucl Med. 1998;25:1277-83.

16. Diehl M, Risse JH, Brandt-Mainz K, Dietlein M, Bohuslavizki $\mathrm{KH}$, Matheja P, et al. Fluorine-18 fluorodeoxyglucose positron emission tomography in medullary thyroid cancer: results of a multicentre study. Eur J Nucl Med. 2001;28:1671-6.

17. Giraudet AL, Vanel D, Leboulleux S, Auperin A, Dromain C, Chami L, et al. Imaging medullary thyroid carcinoma with persistent elevated calcitonin levels. J Clin Endocrinol Metab. 2007;92:418590.

18. Oudoux A, Salaun PY, Bournaud C, Campion L, Ansquer C, Rousseau C, et al. Sensitivity and prognostic value of positron emission tomography with F-18-fluorodeoxyglucose and sensitivity of immunoscintigraphy in patients with medullary thyroid carcinoma treated with anticarcinoembryonic antigen-targeted radioimmunotherapy. J Clin Endocrinol Metab. 2007;92:4590-7.

19. Ong SC, Schoder H, Patel SG, Tabangay-Lim IM, Doddamane I, Gonen M, et al. Diagnostic accuracy of 18F-FDG PET in restaging patients with medullary thyroid carcinoma and elevated calcitonin levels. J Nucl Med. 2007;48:501-7.

20. Rubello D, Rampin L, Nanni C, Banti E, Ferdeghini M, Fanti S, et al. The role of $18 \mathrm{~F}-\mathrm{FDG} \mathrm{PET} / \mathrm{CT}$ in detecting metastatic deposits of recurrent medullary thyroid carcinoma: a prospective study. Eur J Surg Oncol. 2008;34:581-6.

21. Skoura E, Rondogianni P, Alevizaki M, Tzanela M, Tsagarakis S, Piaditis G, et al. Role of [(18)F]FDG-PET/CT in the detection of occult recurrent medullary thyroid cancer. Nucl Med Commun. 2010;31:567-75.

22. Bogsrud TV, Karantanis D, Nathan MA, Mullan BP, Wiseman GA, Kasperbauer JL, et al. The prognostic value of 2-deoxy-2-[18F] 
fluoro-D-glucose positron emission tomography in patients with suspected residual or recurrent medullary thyroid carcinoma. Mol Imaging Biol. 2010;12:547-53.

23. Sundin A, Eriksson B, Bergstrom M, Bjurling P, Lindner KJ, Oberg K, et al. Demonstration of [11C] 5-hydroxy-L-tryptophan uptake and decarboxylation in carcinoid tumors by specific positioning labeling in positron emission tomography. Nucl Med Biol. 2000;27:33-41.

24. Garnett S, Firnau G, Nahmias C, Chirakal R. Striatal dopamine metabolism in living monkeys examined by positron emission tomography. Brain Res. 1983;280:169-71.

25. Reibring L, Agren H, Hartvig P, Tedroff J, Lundqvist H, Bjurling P, et al. Uptake and utilization of [beta-11C]5-hydroxytryptophan in human brain studied by positron emission tomography. Psychiatry Res. 1992;45:215-25.

26. Hoegerle S, Altehoefer C, Ghanem N, Brink I, Moser E, Nitzsche E. 18F-DOPA positron emission tomography for tumour detection in patients with medullary thyroid carcinoma and elevated calcitonin levels. Eur J Nucl Med. 2001;28:64-71.

27. Beheshti M, Pocher S, Vali R, Waldenberger P, Broinger G, Nader $\mathrm{M}$, et al. The value of 18F-DOPA PET-CT in patients with medullary thyroid carcinoma: comparison with 18F-FDG PETCT. Eur Radiol. 2009; 19:1425-34.

28. Beuthien-Baumann B, Strumpf A, Zessin J, Bredow J, Kotzerke J. Diagnostic impact of PET with 18F-FDG, 18F-DOPA and 3-Omethyl-6-[18F]fluoro-DOPA in recurrent or metastatic medullary thyroid carcinoma. Eur J Nucl Med Mol Imaging. 2007;34:16049.

29. Kauhanen S, Seppanen M, Ovaska J, Minn H, Bergman J, Korsoff $\mathrm{P}$, et al. The clinical value of [18F]fluoro-dihydroxyphenylalanine positron emission tomography in primary diagnosis, staging, and restaging of neuroendocrine tumors. Endocr Relat Cancer. 2009;16:255-65.

30. Koopmans KP, de Groot JW, Plukker JT, de Vries EG, Kema IP, Sluiter WJ, et al. 18F-dihydroxyphenylalanine PET in patients with biochemical evidence of medullary thyroid cancer: relation to tumor differentiation. J Nucl Med. 2008;49:524-31.

31. Marzola MC, Pelizzo MR, Ferdeghini M, Toniato A, Massaro A, Ambrosini V, et al. Dual PET/CT with (18)F-DOPA and (18)FFDG in metastatic medullary thyroid carcinoma and rapidly increasing calcitonin levels: Comparison with conventional imaging. Eur J Surg Oncol. 2010;36:414-21.

32. Luster M, Karges W, Zeich K, Pauls S, Verburg FA, Dralle H, et al. Clinical value of $18 \mathrm{~F}$-fluorodihydroxyphenylalanine positron emission tomography/computed tomography (18F-DOPA PET/ CT) for detecting pheochromocytoma. Eur J Nucl Med Mol Imaging. 2010;37:484-93.

33. Pestourie C, Theze B, Kuhnast B, Le Helleix S, Gombert K, Dolle $\mathrm{F}$, et al. PET imaging of medullary thyroid carcinoma in MEN2A transgenic mice using 6-[(18)F]F-L-DOPA. Eur J Nucl Med Mol Imaging. 2010;37:58-66.

34. Luster M, Karges W, Zeich K, Pauls S, Verburg FA, Dralle H, et al. Clinical value of 18 -fluorine-fluorodihydroxyphenylalanine positron emission tomography/computed tomography in the follow-up of medullary thyroid carcinoma. Thyroid. 2010;20:527-33.
35. Kauhanen S, Schalin-Jantti C, Seppanen M, Kajander S, Virtanen $\mathrm{S}$, Schildt J, et al. Complementary roles of 18F-DOPA PET/CT and $18 \mathrm{~F}-\mathrm{FDG}$ PET/CT in medullary thyroid cancer. J Nucl Med. 2011;52:1855-63.

36. Marzola MC, Ferdeghini M, Rampin L, Banti L, Grassetto G, Cittadin S, et al. Sequential 18F-FDG and 18-F-DOPA PET/CT in recurrent medullary thyroid cancer (MTC). J Nucl Med. 2010;51 Suppl 2:71.

37. Srirajaskanthan R, Kayani I, Quigley AM, Soh J, Caplin ME, Bomanji J. The role of $68 \mathrm{Ga}$-DOTATATE PET in patients with neuroendocrine tumors and negative or equivocal findings on 111In-DTPA-octreotide scintigraphy. J Nucl Med. 2010;51:875-82.

38. Reubi JC, Chayvialle JA, Franc B, Cohen R, Calmettes C, Modigliani E. Somatostatin receptors and somatostatin content in medullary thyroid carcinomas. Lab Invest. 1991;64:567-73.

39. Papotti M, Kumar U, Volante M, Pecchioni C, Patel YC. Immunohistochemical detection of somatostatin receptor types 1-5 in medullary carcinoma of the thyroid. Clin Endocrinol Oxf. 2001;54:641-9.

40. Iten F, Muller B, Schindler C, Rochlitz C, Oertli D, Macke HR, et al. Response to [90Yttrium-DOTA]-TOC treatment is associated with long-term survival benefit in metastasized medullary thyroid cancer: a phase II clinical trial. Clin Cancer Res. 2007;13:6696-702.

41. Jang HW, Choi JY, Lee JI, Kim HK, Shin HW, Shin JH, et al. Localization of medullary thyroid carcinoma after surgery using (11)C-methionine PET/CT: comparison with (18)F-FDG PET/CT. Endocr J. 2010;57:1045-54.

42. Dackiw AP. The surgical management of medullary thyroid cancer. Otolaryngol Clin North Am. 2010;43:365-74.

43. Tai D, Poon D. Molecular and other novel advances in treatment of metastatic epithelial and medullary thyroid cancers. J Oncol. 2011;2011:pii 398564

44. Chen H, Sippel RS, O'Dorisio MS, Vinik AI, Lloyd RV, Pacak K. The North American Neuroendocrine Tumor Society consensus guideline for the diagnosis and management of neuroendocrine tumors: pheochromocytoma, paraganglioma, and medullary thyroid cancer. Pancreas. 2010;39:775-83.

45. Biermann K, Biersack HJ, Sabet A, Janzen V. Alternative therapeutic approaches in the treatment of primary and secondary dedifferentiated and medullary thyroid carcinoma. Semin Nucl Med. 2011;41:139-48

46. Divgi C. Targeted systemic radiotherapy of pheochromocytoma and medullary thyroid cancer. Semin Nucl Med. 2011;41:369-73.

47. Hu MI. Updates in the management of medullary thyroid cancer. Clin Adv Hematol Oncol. 2011;9:391-4.

48. Wahl RL, Jacene H, Kasamon Y, Lodge MA. From RECIST to PERCIST: evolving considerations for PET response criteria in solid tumors. J Nucl Med. 2009;50 Suppl 1:122S-50S.

49. Walter MA, Benz MR, Hildebrandt IJ, Laing RE, Hartung V, Damoiseaux RD, et al. Metabolic imaging allows early prediction of response to vandetanib. J Nucl Med. 2011;52:231-40.

50. Mian C, Pennelli G, Barollo S, Cavedon E, Nacamulli D, Vianello F, et al. Combined RET and Ki-67 assessment in sporadic medullary thyroid carcinoma: a useful tool for patient risk stratification. Eur J Endocrinol. 2011;164:971-6. 